\title{
Excimer emission in organic-based substitutional solid solutions of metal-organic frameworks
}

\author{
Wesley Newsome wesnewsome@knights.ucf.edu \\ Arnab Chakraborty arnabc@vt.edu \\ Amanda Morris ajmorris@vt.edu \\ Fernando Uribe-Romo_fernando@ucf.edu
}

Excimer emission is a complex phenomenon which deeply relies on stacking of photo-excited organic molecules in solution phase. Furthermore, control of excimer emission has been rarely studied in the solid state and less so in a tunable nature. We present a method to prepare tunable excimer emission in the solid state utilizing organic-based substitutional solid solutions of metalorganic frameworks, MOFs. These materials allow for the sub stoichiometric addition of excimerforming fluorescent links and for the study of its produced emission. By carefully increasing the concentration of an anthracene containing MOF link we can observe an increase in the emission of excimers and tune its quantum yield solely by concentration. These prepared materials are highly crystalline, despite of their varied composition and produce unique photophysical properties due to the large difference in peak emission from the monomer to excimer peak. 